\title{
Descent methods for optimal periodic hereditary control problems
}

\author{
Krystyna Nitka-Styczeń \\ Institute of Engineering Cybernetics, Technical University of Wroctaw \\ Janiszewskiego 11/17, 50-372 Wrockaw, Poland. \\ Tel: 4871202745 . Fax: 4871203408.
}

\begin{abstract}
Continuously operated autonomous processes governed by hereditary state equations are considered. The problem of improving of the optimal steady-state solution by periodic variations of control and state variables is discussed. A new formulation of the periodic hereditary control problem exploiting the heredity shift operator is presented, and its advantages are pointed out. This problem is solved iteratively by descent methods using variable metric ideas and implemented with the help of the Mathematica system. The proposed approach is illustrated by a simple example of ecological hereditary system.
\end{abstract}

\section{Keywords}

Hereditary autonomous process, periodic control problem, heredity shift operator, descent method, Mathematica system.

\section{INTRODUCTION}

Long horizon continuously operated autonomous processes are encountered in many chemical, biochemical, biotechnological, and ecological systems (May, 1973), (Reber, 1979), (Ray, 1981), (Smith and Waltman, 1995). The optimization of such processes is connected with the question if the optimal steady-state solutions can be improved by periodic variations of the state and control variables. Adequate models of many processes under discussion should mirror miscellaneous hereditary phenomena (Abulesz and Lyberatos, 1987), (Colonius, 1988), (Smith and Waltman, 1995). This fact complicates the determination of the optimal periodic control, because necessary optimality conditions formulated as the maximum principle (Colonius, 1988) or as the Banach space optimality conditions (Colonius, 1988) may take a quite complicated form. In particular, the periodic delayed-advanced Hamiltonian system arising from the maximum principle may be unstable and sensitive to parameter changes similarly to the case of undelayed problems, where the appropriate Hamiltonian systems cannot be asymptotically stable according to the Poincare-Lyapunov theorem (Demidovich, 1967). 
For the above reasons descent optimization methods may be helpful in searching of nearly optimal solutions to periodic hereditary control problems. To depict such methods a new formulation of the problem discussed is presented. It uses the process dynamics description in the form of an integral equation equivalent to the basic periodic differential hereditary equation. The equivalent integral equation exploits the so-called heredity shift operator, and acts in the space of continuous functions defined on the process operation period. The simple form of the heredity shift operator (for a simple discrete delay) has been introduced by Krasnosel'skii (1966) in the context of the theory of periodic nonlinear equations.

We generalize the definition of this operator to the case of many lumped and distributed delays, and we adopt it to periodic hereditary control problems. The main advantage of our formulation is connected with the interpretation of periodic admissible trajectories as fixed points of a completely continuous integral operator. Such operators possess high accuracy finite dimensional approximations (Krasnoselskii, 1969). Therefore, the formulation proposed is especially suitable for computational purposes, and in particular for the construction of iterative descent optimization methods.

In the approach considered the control space may be chosen as the Banach space of essentially bounded measurable functions or as the Hilbert space of square-integrable functions. While the first choice is useful for the analysis of optimality conditions, the second one may be advantageously exploited to design enhanced descent optimization directions (Nitka-Styczeń, 1995).

We use the following spaces of $n$-dimensional functions defined on the interval $(\alpha, \beta)$ : $C^{0, n}(\alpha, \beta)$ the space of continuous functions, $L_{p}^{n}(\alpha, \beta)$ the space of $p$ th power integrable functions, $W_{p}^{1, n}(\alpha, \beta)$ the space of differentiable functions with derivative in $L_{p}^{n}(\alpha, \beta)$.

\section{OPTIMAL PERIODIC HEREDITARY CONTROL PROBLEM}

Consider the following optimal periodic hereditary control (OPHC) problem: minimize the time-averaged objective function

$J(\tau, x, u)=\frac{1}{\tau} \int_{0}^{\tau} g(x(t), u(t)) d t$

subject to

$\dot{x}(t)=f\left(x_{t}, u(t)\right), \quad t \in[0, \tau]$,

$x_{0}=x_{\tau}$,

$\frac{1}{\tau} \int_{0}^{\tau} \phi(x(t), u(t)) d t=0$

$\tau \in \mathcal{T}, \quad u(t) \in U, \quad t \in[0, \tau]$,

where $\tau$ is the operation period, $\mathcal{T}=\left[\tau^{0}, \tau^{1}\right] \subset R_{+}, r \ll \tau_{0}, x \in \mathcal{X}=W_{p}^{\mathbf{1}, n}(0, \tau)$ is the momentary state trajectory, $x_{t} \in \mathcal{X}_{0}=W_{p}^{1, n}(-r, 0)$ is the complete state at the time $t$, $u \in \mathcal{U}=L_{p}^{m}(0, \tau)$ is the control, $U$ is a (bounded and closed) parallelepiped in $R^{m}$, and

$g: R^{n} \times R^{m} \rightarrow R^{1}, \quad \phi: R^{n} \times R^{m} \rightarrow R^{\nu}$, 


$$
\begin{aligned}
& f: \mathcal{X}_{0} \times R^{m} \rightarrow R^{n}, \\
& f\left(x_{t}, u(t)\right)=\tilde{f}\left(\xi_{0}, \ldots, \xi_{q}, \xi_{q+1}, \ldots, \xi_{q+\tilde{q}}, u(t)\right), \quad \tilde{f}: R^{n \times(1+q+\tilde{q})} \rightarrow R^{n}, \\
& \xi_{h}=x\left(t-r_{h}\right), \quad h=0,1, \ldots, q ; \quad 0=r_{0}<r_{1}<\ldots<r_{q} \leq r, \\
& \xi_{q+h}=\int_{-\tilde{r}_{h}}^{0} \Gamma_{h}(\theta) x(t+\theta) d \theta, \quad \Gamma_{h}: R \rightarrow R^{n \times n}, \quad h=1, \ldots, \tilde{q}, \quad 0<\tilde{r}_{1}<\ldots<\tilde{r}_{\tilde{q}} \leq r .
\end{aligned}
$$

Thus we deal with the case of many lumped and distributed delays.

Assumption 1 The functions $g, \tilde{f}, \phi$ are continuously differentiable, and the functions $\Gamma_{h}$ are continuous.

Together with the OPHC problem we consider the following optimal integral hereditary control (OIHC) problem: minimize

$J(\tau, x, u)=\frac{1}{\tau} \int_{0}^{\tau} g(x(t), u(t)) d t$

subject to

$x(t)=x(\tau)+\int_{0}^{t} \tilde{f}\left(S_{\theta} x, u(\theta)\right) d \theta, \quad t \in[0, \tau]$,

$\frac{1}{\dot{\tau}} \int_{0}^{\tau} \phi(x(t), u(t)) d t=0$,

$\tau \in \mathcal{T}, \quad u(t) \in U, \quad t \in[0, \tau]$,

where $x \in \tilde{\mathcal{X}}=C^{0, n}(0, \tau), u \in \mathcal{U}=L_{p}^{m}(0, \tau)$, and the heredity shift operator

$S_{t}: \tilde{\mathcal{X}} \rightarrow R^{n \times(1+q+\tilde{q})}$

is defined as follows:

$S_{t} x=\left(S_{t, h} x\right)_{h=0,1, \ldots, q+\tilde{q}}$,

$S_{t, h} x=\left\{\begin{array}{ll}x\left(t-r_{h}\right) & \text { if } \quad r_{h} \leq t \leq \tau, \\ x\left(\tau+t-r_{h}\right) & \text { if } 0 \leq t<r_{h},\end{array} \quad h=0,1, \ldots, q\right.$

$S_{t, h} x=\int_{-t}^{0} \Gamma_{h}(\theta) x(t+\theta) d \theta+\int_{-r_{h}}^{-t} \Gamma_{h}(\theta) x(\tau+t+\theta) d \theta, \quad p=q+1, \ldots, q+\tilde{q}$.

Lemma 1 The problems OPHC and OIHC are equivalent.

The proof follows from the fact that the set of solutions $(x, u)$ of the equations $(2)$ and (3) and the set of solutions $(x, u)$ of the equation (7) coincide. 
Lemma 2 The operator $A: \tilde{\mathcal{X}} \rightarrow \tilde{\mathcal{X}}$ defined as $(A x)(t)=x(\tau)+\int_{0}^{t} \tilde{f}\left(S_{\theta} x, u(\theta)\right) d \theta, \quad t \in[0, \tau]$,

is completely continuous for every admissible control $u$.

The proof follows from the fact that the heredity shift operator transforms every equibounded set $X$ in $\tilde{\mathcal{X}}$ into an equibounded one in the same space. This allows one to show under Assumption 1 equiboundedness and equicontinuity of the functions (10) on the set $X$.

Thus the convertion of the OPHC problem into the OIHC problem is justified by the simplicity of the latter problem taking the form of controlled fixed points of the complete continuous operator $A$.

\section{DESCENT METHOD IN THE PERIOD AND CONTROL SPACE}

We assume that the additional constraints (4) an (5) are handled by penalty or/and projection methods. We consider at first the possibility of the local reduction of the OIHC problem to the control space for fixed period $\tau$.

This reduction allows one to construct a descent sequence, for which a high accuracy resolution of the periodic hereditary process equation is preserved.

We write the process equation $(7)$ as the equality $P(\tau, x, u)=0$, where the nonlinear operator $P: R \times \tilde{\mathcal{X}} \times \mathcal{U} \rightarrow \tilde{\mathcal{X}}$ is defined as

$P(\tau, x, u)(t)=x(t)-x(\tau)-\int_{0}^{t} \tilde{f}\left(S_{\theta} x, u(\theta)\right) d \theta, \quad t \in[0, \tau]$.

Assumption 2 The unity does not belong to the spectrum of the operator $A: \tilde{\mathcal{X}} \rightarrow \tilde{\mathcal{X}}$.

This assumption guarantees that the F-derivative $P_{x}: \tilde{\mathcal{X}} \rightarrow \tilde{\mathcal{X}}$ defined by the formula

$\left(P_{x} \delta x\right)(t)=\delta x(t)-\delta x(\tau)-\int_{0}^{t} \tilde{f}_{x}\left(S_{\theta} x, u(\theta)\right) \delta x(\theta) d \theta, \quad t \in[0, \tau]$

where $\delta x \in \tilde{\mathcal{X}}$, is continuously invertible.

Lemma 3 The OIHC problem is under Assumption 2 locally reducible for fixed period $\tau$ to the control space $\mathcal{U}$, i.e. there exists a uniquely determined function $x(\tau, u)$ solving the equation (7) in the vicinity of the control $u$ with fixed parameter $\tau$.

The proof is a consequence of the standard implicit function theorem.

Let us denote the objective function reduced to the period and control space as $\tilde{J}(\tau, u)=J(\tau, x(\tau, u), u)$.

Then the differential of the functional $\tilde{J}$ can be written in the form

$\tilde{J}_{u} \delta u=\frac{1}{\tau} \int_{0}^{\tau}\left(g_{x}(x(t), u(t))\left(x_{u}(\tau, u) \delta u\right)(t)+g_{u}(x(t), u(t)) \delta u(t)\right) d t$, 
where $x_{u}(\tau, u) \delta u=P_{x}^{-1} P_{u} \delta u$. The formula (13) can be used to develop the following principal descent optimization algorithm for the OIHC problem:

\section{Algorithm 1}

1. Choose a triple $(\tau, x, u)$.

2. Solve the process equation (7) for a given $(\tau, u)$ with the initial approximations $x$ by means of the Newton algorithm

$$
x_{+}=x-\delta x, \quad P_{x}(\tau, x, u) \delta x=P(\tau, x, u) .
$$

3. Compute an improved control

$u_{+}=u-\alpha_{+} D \tilde{J}_{u}^{*}$,

where $D \tilde{J}_{u}^{*}$ is a descent direction determined by a positive operator $D$ and gradient $\tilde{J}_{u}$, while $\alpha_{+}$is a line search coefficient.

4. Repeat 3. to satisfy the stop criterion

$\left\|\tilde{J}_{u}\right\|_{u} \leq \epsilon$

5. Go to 1 .

\section{COMPUTATIONAL IMPLEMENTATION}

To make the principal algorithm computationally implementable we approximate the control by step functions and the state by roof functions

$u(t)=\sum_{k=1}^{K} e_{k}(t) u_{k}, \quad x(t)=\sum_{k=0}^{K} \psi_{k}(t) x_{k}$.

The choice of the roof basis (Daniel 1971, Wierzbicki 1984) for the state is justified by its compatibility with the control approximation.

We denote by the upper index $K$ operators and functions discretized on the time grid $t_{k}=\frac{\tau}{K} k, k=1, \ldots, K$

$x^{K}=\operatorname{vec}_{k=1}^{K} x\left(t_{k}\right)=\operatorname{vec}_{k=1}^{K} x_{k}$.

The discretized version of the shift operator may be written as follows:

$\tilde{S}_{k}: R^{n \times K} \rightarrow R^{n \times(1+q+\tilde{q})}$

is defined as follows

$\tilde{S}_{k} x^{K}=\left(\tilde{S}_{k, h} x^{K}\right)_{h=0, \ldots, q+\tilde{q}}$ 


$$
\begin{aligned}
& \tilde{S}_{k, h} x^{K}=\left\{\begin{array}{ll}
x_{k-\left[r_{h} \frac{\tau}{K}\right]}, & \text { for } k-\left[r_{h} \frac{\tau}{K}\right] \geq 0 \\
x_{K+k-\left[r_{h} \frac{\tau}{K}\right]}, & \text { for } k-\left[r_{h} \frac{\tau}{K}\right]<0
\end{array} \quad h=0, \ldots, q,\right. \\
& \tilde{S}_{k, h} x^{K}= \begin{cases}\sum_{j=k-\left[r_{h} \frac{\tau}{K}\right]+1}^{k} \Gamma_{j-k} x_{j} & \text { for } k-\left[r_{h} \frac{\tau}{K}\right] \geq 0 \\
\sum_{j=1}^{k} \Gamma_{j} x_{j} & \text { for } k-\left[r_{h} \frac{\tau}{K}\right]<0 \\
+\sum_{j=K+k-\left[r_{h} \frac{\tau}{K}\right]+1}^{K} \Gamma_{j-K-k} x_{j} & h=q+1, \ldots, q+\tilde{q},\end{cases}
\end{aligned}
$$

where $[z]$ denotes integer of $z$ closest to $z$.

The solution of the integral hereditary state equation (7) by the Newton method given by the formulas (14) can be written in the discretized version

$$
\begin{aligned}
& x_{k+}=x_{k}-\delta x_{k}, \quad k=1, \ldots, K, \\
& \delta x_{k}-\delta x_{K}-\sum_{h=0}^{q+\tilde{q}} \sum_{j=1}^{k} \frac{\tau}{K} \tilde{f}_{\xi_{h}}\left(\tilde{S}_{j} x^{K}, u_{j}\right) S_{j, h} \delta x^{K}=x_{k}-x_{K}-\sum_{j=1}^{k} \frac{\tau}{K} \tilde{f}\left(S_{j} x^{K}, u_{j}\right) .
\end{aligned}
$$

We compose these equations in the matrix form encompassing all discrete time moments, and we write the second step of the principal descent optimization algorithm as

$$
x_{+}^{K}=x^{K}-\delta x^{K}, \quad \delta x^{K}=\left[I-J-\sum_{h=0}^{q+\tilde{q}} \tilde{F}_{\xi_{h}}^{K} M_{h}^{K}\right]^{-1} \mathcal{P}^{K},
$$

where $I$ is the identity matrix, $J$ is the matrix with the elements equal to 1 in the last column and others equal to $0, \tilde{F}_{\xi_{h}}^{K}$ is lower diagonal matrix with elements $\frac{\tau}{K} \tilde{f}_{\xi_{h}}\left(S_{j} x^{K}, u_{j}\right)$ below the diagonal in the $j$ th column,

$$
M_{h}^{K} \delta x^{K}=\operatorname{vec}_{j=1}^{K} S_{j, h} \delta x^{K}, \quad \mathcal{P}^{K}=\operatorname{vec}_{k=1}^{K}\left[x_{k}-x_{K}-\sum_{j=1}^{k} \frac{\tau}{K} \tilde{f}\left(S_{j} x^{K}, u_{j}\right)\right] .
$$

We also write the gradient (13) in the discretized version

$$
\tilde{J}_{u^{K}}=\frac{1}{K}\left(\mathcal{G}_{x}^{K}\left[I-J-\sum_{h=0}^{q+\tilde{q}} \tilde{F}_{\xi_{h}}^{K} M_{h}^{K}\right]^{-1} \mathcal{P}_{u}^{K}+\mathcal{G}_{u}^{K}\right)
$$

where $\mathcal{P}_{u}^{K}$ is the lower diagonal matrix with the elements $\frac{\tau}{K} \tilde{f}_{u}\left(\tilde{S}_{j} x^{K}, u_{j}\right)$ in the $j$ th column, $\mathcal{G}_{x}^{K}=\left[g_{x}\left(x_{1}, u_{1}\right), \ldots, g_{x}\left(x_{K}, u_{K}\right)\right], \mathcal{G}_{u}^{K}=\left[g_{u}\left(x_{1}, u_{1}, \ldots, g_{u}\left(x_{K}, u_{K}\right)\right]\right.$.

The convergence of the discretized optimization method is connected with the convergence of the process equation approximation, the approximation of its derivative with respect to the control, and with the convergence of the approximate integration formula for the objective function. The two first question can be analysed, due to the complete continuity of the operator $A$, by applying the Banach-Steinhaus theorem in the form (Krasnosel'skii, 1969)

$\left\|\mathcal{A}^{K}\right\| \leq c, \quad K=K_{0}, K_{0}+1, \ldots$, 
$\lim _{K \rightarrow \infty}\left\|E_{K} A x-\mathcal{A}^{K} E_{K} x\right\|=0, \quad E_{K} x=\operatorname{vec}_{k=0}^{K} x\left(t_{k}\right)$.

Next the convergence of nearly optimal solutions for discretized problems can be analysed, for example, along the lines of Daniel (1971).

The computational algorithm (15) and (16) with the variable metric matrix has been applied with the help of the Mathematica system to the following example of the OIHC problem.

Example 1 Consider the following OPHC control problem for a single-species population model with time lag: minimize

$J(\tau, x, u)=-\frac{1}{\tau} \int_{0}^{\tau} u(t) x(t) d t$

subject to

$\dot{x}(t)=c x(t)\left(1-\frac{x(t-r)}{a}\right)-u(t) x(t)$,

$u(t) \in\left[0, u^{\max }\right]$.

This problem corresponds to the maximization of the harvesting effect with the help of the periodic operation (optimal periodic harvesting problem).

We assume the following parameter values $c=1.3797, a=3.8187, \quad r=2.2764$. Then the optimal steady-state process has been computed $\stackrel{\circ}{x}=1.9093, \stackrel{\circ}{u}=0.6898, \quad J\left(\frac{o}{\bar{x}}, \stackrel{\circ}{u}\right)=$ -1.3172 .

The II form (Colonius, 1988) has the following expression

$\Pi(\omega)=\frac{a c \cos (\omega r)}{c^{2} / 2+2 \omega^{2}-2 \omega \omega \sin (\omega r)}$.

It follows from the shape of the curve $\Pi(\omega)$ that the choice of the operation frequency has considerable influence on the effectiveness of periodic control. The periodic operation should be especially advantageous for the $\omega=0.6900$ i.e. for the period $\tau=9.1059$.

Starting from the steady control with harmonic variation, and from the steady state, after three steps of the algorithm we have obtained suboptimal periodic control process shown in Figure 1, with the cost function $J=-2.700$.

The main advantage of the proposed approach consists in the simplicity of the computation of periodic solutions to the hereditary state equation. A fast convergence of the Newton method applied to this end has been observed. The disadvantage of the method consists in the necessity of applying of small control steps to guarantee the stability of the Newton method.

The suboptimal control computed may be used for final optimization performed with the help of the maximum principle.

The paper carried out within the research project Nr 8 S505 01107 of Polish State Committee for Scientific Research 


\section{$u(t)$}

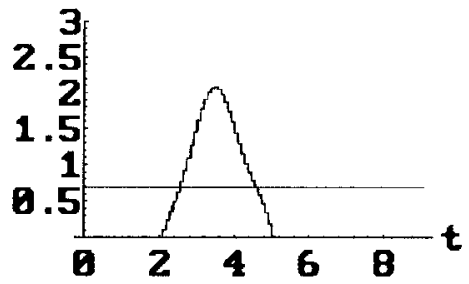

$x(t)$

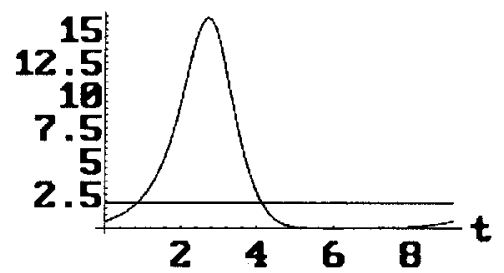

Figure 1 The steady-state process and suboptimal periodic process for the Example 1

\section{REFERENCES}

Abulesz, E.M. and Lyberatos, G. (1987) Periodic optimization of continuous microbial growth processes. Biotechnology and Bioengineering, 29, 1059-65.

Colonius, F. (1988) Optimal Periodic Control. Lecture Notes in Mathematics Nr 1313. Springer-Verlag, Berlin.

Daniel, J.W. (1971) The Approximate Minimization of Functionals. Prentice Hall, Englewood Cliffs, New Jersey.

Demidovich, B.P. (1967) Lectures on Mathematical Stability Theory. Nauka, Moscow.

Krasnosel'skii, M. (1966) The Shift Operator Along the Trajectories of Differential Equations. Nauka, Moscow (in Russian).

Krasnosel'skii, M. (1969) The Approximate Solving of Operator Equations. Nauka, Moscow (in Russian).

May, R.M. (1973) Stability and Complexity in Model Ecosystems. Princeton, University Press, Princeton, N.J.

Nitka-Styczen, K. (1995) Optimal Retarded Control Problems with Free Initial States: A Descent Method. System Analysis Modelling Simulation (to appear).

Ray, W.H. (1981) Advanced Process Control. Mc Grow Hill, New York.

Reber, D.C. (1979) A finite difference technique for solving optimization problems governed by linear functional differential equations. Journal of Differential Equations, 32, 193-232.

Smith, H.L. and Waltman, P. (1995) The Theory of the Chemostat. Dynamics of Microbial Competition. Cambridge University Press, Cambrigde.

Wierzbicki, A. (1984) Models and Sensitivity of Control Systems. Elsevier, Amsterdam.

Wolfram Research, Inc. (1994) Mathematica. A System for Doing Mathematica by Computer. Wolfram Research, Inc., Champaign, Illinois. 